\section{Stop making sense}

DURING mitosis, the chromosomes in the nucleus of a cell move towards the poles of the mitotic spindle, a scaffold of microtubules, in preparation for nuclear and cell division. R. Margolis and collaborators have identified a protein from brain that stabilizes the dynamic structure of the spindle and may generate the movement of chromosomes to the spindle's poles in vivo (EMBO J. 9 , $4095-4102 ; 1990)$. The protein, called 'stop' (stable tubule only polypeptide), is known to stabilize microtubules in vitro.

The new work shows that it is tightly associated with microtubules in vitro during three cycles of assembly and disassembly. The authors also show that, under physiological conditions, stop is preferentially distributed only on those microtubules thought to move chromosomes to the poles in anaphase. The next step is to see if stop could have this function in cells other than neurons.

\section{See-through ice}

ANTARCTIC sea ice is at its most transparent in the spring (September-November), just when ozone depletion allows the highest fluxes of solar ultraviolet radiation, according to $\mathrm{H}$. J. Trodahl and R. G. Buckley (Geophys. Res. Lett. 17, 2177 $2179 ; 1990)$. Concerned by the deleterious effect of increased ultraviolet levels on Antarctic ecology, the authors monitored the ice's transparency using an artificial light source. They estimate that the severe ozone holes of 1987 and 1989 would have caused a tenfold increase in the dose of ultraviolet to algae below the ice. The result, they say, is that the Antarctic ecosystem must have been affected, as these algae are highly sensitive to this radiation.

\section{Flipping lipids}

THE inner and outer leaflets of the plasma membranes of red cells - and many others - have different lipid compositions. The asymmetry is maintained by an ATP-dependent translocase. The hunt for this enzyme has converged recently on the Rhesus blood group protein. A.J. Schroit et al. (Biochemistry 29, 10303-10306; 1990) have now shown that a protein, which migrates in gels with the Rhesus polypeptide, is labelled with a lipid affinity analogue or with an inhibitor of translocation, and the labelled molecule is precipitated with anti-Rhesus antibody. So far so good - but both Schroit et al. and R.E. Smith and D.L. Daleke (Blood 76, 1021-1027; 1990) have looked at $\mathrm{Rh}_{\text {null }}$ cells, which are devoid of the Rhesus protein, and found that they translocate lipids just like normal cells. This leads to a fine philosophical antithesis, for Smith and Daleke conclude that the translocase cannot be the Rhesus protein and Schroit et al. that the Rhesus protein must be present in $\mathrm{Rh}_{\text {null }}$ cells in some new. antigenically silent phenotype.

\title{
The solar-stellar connection
}

\section{Mark S. Giampapa}

THE 11-year solar cycle, it is currently held, has just passed its maximum - a maximum more intense than seen for many decades. But how big is the range of physical behaviour that the Sun can go through? The 'Maunder minimum', a period of quiescence between 1650 and 1730 , shows that activity outside the present range is possible. On page 520 of this issue', Baliunas and Jastrow tackle this question by looking at the range of activity apparent on other stars that are of the same type as the Sun. Given the Sun's influence on our climate, their evidence for deep Maunder-minimum-type quiescence is particularly interesting.

The Sun's atmosphere reveals a variety of bright features. They originate from magnetic fields generated deep in the solar interior. Dynamo processes ${ }^{2}$ are presumed to give rise to the intense chromospheric and coronal emissions which often coincide with the sites of magnetic field structures. Stars also show these characteristics, although there can be important differences in the scale of activity associated with emergent magnetic flux. Just as detailed study of the Sun serves as a guide to stellar magnetism, so the parallel investigation of stellar analogues of solar magnetic activity yields tests of theories developed purely within a solar context. Quantities such as rotation rate, depth of the convection zone, surface gravity and effective temperature and evolutionary status can take a wide range of non-solar values, stretching models well beyond any familiar territory.

Although the energy residing in solar and stellar surface magnetic fields is negligible compared to the energy associated with total luminosity (as is the radiant energy of the associated hot atmospheric layers above the photosphere that we identify as the chromosphere and corona), the dynamical role the fields play is significant. In particular, magnetic fields determine the angular-momentum evolution of the late-type stars and modulate their total radiative output. In the case of the Sun, this leads to observable changes in the total irradiance.

As demonstrated by Baliunas and Jastrow, solar-type stars can undergo a much wider range of cyclical variation than is known for the Sun. The interpretation of these chromospheric variations within the context of changes in magnetic activity relies on the assumption that chromospheric emission features are correlated with surface magnetic flux. This is well-established in the case of the Sun ${ }^{3}$. Direct evidence has recently emerged that this is also true for solar-like stars. In particular, enhanced levels of chromo- spheric and coronal emission in solar-type stars are correlated with high fractional coverage (or filling factor) of magnetic field $^{+}$. Thus, high intensities of chromospheric $\mathrm{Ca}$ II $\left(\mathrm{Ca}^{+}\right)$emission correspond to high levels of surface magnetic flux. The direct correlation of luminosity variability in solar-type stars with changes in chromospheric emission not only corroborates the detection of subtle variability in the solar irradiance but strongly suggests that the range of solar variability can be larger The potential range depends on the level of magnetic flux present at each phase of the solar cycle.

Another of Baliunas and Jastrow's results, indicating that solar-type stars have extended periods of dormancy in magnetic activity, is particularly exciting. The historical record ${ }^{6}$ suggests that there have been periods, such as the Maunder minimum, of abnormally low levels of magnetic activity on the Sun in the past, but the conclusion is controversial. When these stellar observations are combined with the extant ${ }^{1+} \mathrm{C}$ terrestrial record', another indicator of solar activity, the evidence for episodes of nearly complete solar quiescence becomes quite persuasive. Moreover, Baliunas and Jastrow find that the level of chromospheric emission in quiescent stars is even lower than that observed during the minimum phase of the modern solar cycle. The results tentatively suggest that solar-like stars spend prolonged periods in these minima. In view of the correlation of brightness changes with the level of magnetic activity as seen in both solar and stellar data, the impact of a Maunder-minimum phase on the terrestrial climate could be significant.

A comparison of the strength of the solar $\mathrm{Ca}$ II line emission strength as seen in full disk observations and in centreof-the-disk observations (see figure) confirms that the disk-integrated $\mathrm{Ca}$ II $\mathrm{K}$ line of the Sun (the Sun seen as a star) exhibits the 11-year solar cycle, but that variations at disk centre do not. Thus, the cyclic variability in radiative proxies of magnetic activity must arise from localized concentrations of magnetic flux rather than from

Baliunas, \& Jastrow. Nature 348, 520-523 (1990)

2. Vaiana, G. S. \& Rosner, R. A. Rev. Astr. Astrophys. 16. $393-428$ (1978).

. Skumanich, A. et al. Astrophys. J. 200, 747-763(1975)

4. Saar, S. H. in The Sun in Time (eds Sonett, C. P. Giampa pa, M. S. \& Matthews, M. S.) (University of Arizona Press, Tucson, in the press).

5. Radick, R. R. in The Sun in Time (eds Sonett, C. P., Giampapa, M. S. \& Matthews. M. S.) (University of Arizona Press. Tucson, in the press)

6. Eddy, J. A. in The Solar Output and its Variation (ed. White, O. R.) 51-71 (Colorado Associated University Press, Boulder, 1977

7. Damon, P. E. in The Solar Output and its Variation (ed White, O. R.) $429-448$ (Colorado Associated University Press, Boulder, 1977). 